\title{
MARIA THERESIA UND DIE ZENSUR
}

\author{
NORBERT BACHLEITNER
}

\author{
Universität Wien ${ }^{1}$ \\ Norbert.bachleitner@univie.ac.at
}

\begin{abstract}
Im Jahr 1751 führte Maria Theresia als Teil ihrer Reformen erstmals die systematische Zensur im Habsburger Reich ein. Eine Kommission, bestehend aus ihrem Leibarzt Gerard van Swieten und anderen Gelehrten überprüften sämtliche Manuskripte, die aus dem Ausland kamen. Verbotene Bücher wurden in einem Index geführt (Catalogus librorum prohibitorum), der bis zu ihrem Ableben, 1780, regelmäßig publiziert wurde. Die Zensur war eher tolerant und versuchte im Sinne einer moderaten Aufklärung zu agieren. Kritik an der katholischen Religion war jedoch unmöglich. Aristokraten, Gelehrte und Mitglieder der höheren Bourgeoisie bekamen die Erlaubnis, verbotene Bücher zu lesen, die Zensur anerkannte und befürwortete nämlich die Akzeptanz gesellschaftlicher Unterschiede. Auch wenn man unter Maria Theresia von einer toleranten Zensur sprechen kann, so war sie doch strenger als unter Joseph II., ihrem Sohn. Die meisten der verbotenen Bücher erschienen auf Deutsch, aber mehr als ein Drittel der Werke war auf Französisch geschrieben worden. Auch lateinische Bücher wurden in großer Zahl verboten. Der am häufigsten verbotene Autor war Voltaire und unter den top-ten der verbotenen Franzosen finden wir Namen wie d'Argens, Rétif de la Bretonne, Crébillon fils, sowie Repräsentanten der aufklärerischen Philosophie, wie Friedrich II., Rousseau und Georg Friedrich Meier.
\end{abstract}

Schlüsselwörter: Zensur, Maria Theresia, Gerard van Swieten, Aufklärung, Protestantismus, Statistik verbotener Bücher, Bücherverbrennung, verbotene Autoren.

\section{Die Vorgeschichte}

Als Folge der Erfindung des Buchdrucks und der wachsenden Buchproduktion sowie der Reformation wurde im deutschsprachigen Raum die Zensur eingeführt. Basis des kaiserlichen Hoheitsrechts über das Buch- und Pressewesen war das sogenannte Bücherregal, ein Monopol, das der Kaiser später mit den Landesherren teilte. Damit verbunden war das Recht auf Verleihung von Druckprivilegien (Privilegia impressoria), das Autoren und/oder Verleger vor unberechtigtem Nachdruck schützte. Mit der Sanctio pragmatica von 1623 wurde die Zensur in (Nieder-)Österreich der Universität Wien übertragen. Da die Jesuiten in den katholischen Ländern die Mehrzahl der Lehrkanzeln für Theologie und Philosophie besetzten, übten sie die Zensur von Manuskripten und Büchern in diesen Disziplinen aus, was äußerste Strenge gegenüber protestantischen Schriften bedeutete. 
Die Kirche und die weltlichen Regierungen teilten sich also fortan die Zensuraufgaben, ohnehin herrschte das religiöse Schrifttum bis weit ins 18. Jahrhundert hinein auf dem Buchmarkt vor, das wichtigste politische Anliegen war die Wahrung des Religionsfriedens. In Österreich bedeutete das vor allem die Fernhaltung von ,sectischen', d. h. protestantischen Schriften.

Die Maßnahmen zur Verhinderung der Verbreitung protestantischer Schriften umfassten die Kontrolle der Kolporteure (,Bücherträger'), die einen Pass des Religionskonzesses, einer landesfürstlichen Behörde, erwerben und ihre Ware genehmigen lassen mussten (vgl. Scheutz 2014). In Böhmen mit seiner ursprünglich zu achtzig bis neunzig Prozent protestantischen Bevölkerung und entsprechend radikaler Zwangsrekonfessionalisierung stand nach dem Sieg Ferdinands II. in der Schlacht am Weißen Berg 1620 bis zu Josephs Toleranzpatent von 1781 auf Handel mit verbotenen Büchern die Todesstrafe, die allerdings vermutlich nur selten vollstreckt wurde (s. Ducreux 2005).

Da sich eine systematische Überwachung des Bücherverkehrs weder im religiösen noch im politischen Segment gewährleisten ließ, beschränkten sich die staatlichen Maßnahmen auf die symbolische Geste der Verbrennung eines Exemplars einer Schrift, das stellvertretend für den Verfasser bzw. den Geist des Werks verbrannt wurde. Gelegentlich wurden, um die Nachhaltigkeit der Maßnahme zu steigern, das Buch und sein Autor zusammen verbrannt (vgl. Rafetseder 1988). Das in der Vernichtung durch Feuer implizierte Pathos und die Vorstellung einer dadurch gegebenen direkten Verbindung mit höheren Mächten wird in dem Titelkupfer des Römischen Index in der Ausgabe von 1711 deutlich: Der Heilige Geist sendet hier den Geistlichen (Zensoren?) die Energie, die sie nur reflektieren und damit das die Bücher als Träger des Bösen vernichtende Feuer entfachen (vgl. Abbildung 1).

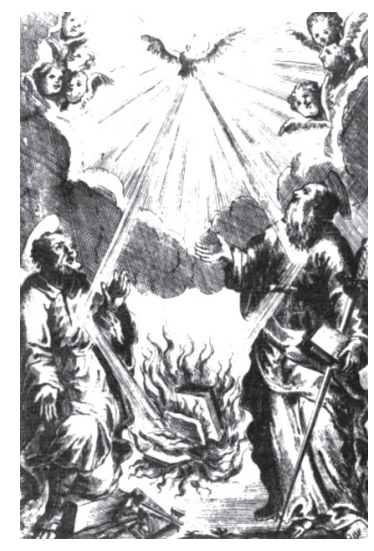

Abbildung 1:

Titelkupfer des päpstlichen Index librorum prohibitorum von 1711 
Erst in einem kaiserlichen Edikt von 1715 wurden erstmals explizit auch politische und Schmähschriften, die die Regierung und die Gesetze des Hl. Römischen Reiches sowie einzelne Persönlichkeiten angriffen, erwähnt (s. Fischer 1982, 38). Der Umstand, dass die Theologie auf dem Buchmarkt an Boden zu verlieren begann und vermehrt auch die weltliche Herrschaft diskutiert wurde, zog eine Verschiebung der Zensurkompetenzen hin zum Staat nach sich. Zudem fühlte sich der Staat zunehmend auch für das Seelenheil der Untertanen zuständig. Da die geistlichen Instanzen, d. h. in erster Linie der Papst, die Bischöfe und die Jesuiten an den Universitäten, diese Kompetenz nicht freiwillig aufgaben, kam es zu einer das gesamte 18. Jahrhundert andauernden Auseinandersetzung um die Zensurmacht. Die mit der Gewährung von Druckprivilegien verbundene Durchsicht von Manuskripten lag noch immer in der Hand der Universität, die Kontrolle des Buchhandels in Form von Visitationen von stationären Buchhandlungen und den Märkten sowie der Büchereinfuhr an den Grenzen wurde zum Teil von der Universität, zum Teil vom Staat wahrgenommen. Zu diesem Zweck wurden von den Landesregierungen Bücherrevisionskommissionen eingerichtet, und zwar 1723 in Prag für Böhmen und 1732 in Graz für Innerösterreich (Steiermark).

1748 tauchte in Prag eine Aufsehen erregende Schrift auf, und zwar die unter dem Pseudonym Rochezang von Isecern publizierte Historische und Geographische Beschreibung des Königreiches Böheim. Sie enthielt eine kritische Beleuchtung der Verleihung der böhmischen Stimme bei der Wahl Karls VII. zum Kaiser an Maria-Theresia, deren Wahlrecht sehr umstritten war, sowie Berichte über die aktuellen Kriegsaktivitäten. Da die Stimmung in Böhmen aufgeheizt war und man insbesondere Bauernunruhen befürchtete, wurde das Buch im November 1749 in Wien verbrannt und der Name des Verfassers am Galgen angeschlagen. In Wien tauchte kurz darauf ein Buch mit dem Titel Lettres d'un Seigneur Hollandois à un de ses amis auf, das Maria-Theresias Erbrecht infrage stellte (vgl. Fournier 1876, 403-404). Alle diese Fälle mussten individuell behandelt und die Urteile durch Dekrete verkündet werden, was ein sehr schwerfälliges Verfahren bedeutete. Der Handlungsbedarf bezüglich der Einsetzung eines effizienten Zensursystems verdichtete sich. Darüber hinaus ist die Einrichtung moderner Verwaltungsstrukturen in den absoluten Monarchien um die Mitte des 18. Jahrhunderts allerseits, zum Beispiel auch in Frankreich und den deutschen Staaten, zu beobachten. Eine solche moderne Bürokratie schloss regelmäßig ein zensorisches Überwachungssystem ein, das Arbeitsteilung und Professionalität, ein Reglement, das das Verfahren kodifizierte, und Aktendokumentation umfasste. Auch die in Österreich zu beobachtende Verdrängung der kirchlichen Institutionen aus den Zensurvorgängen waren fixer Bestandteil dieser Bürokratie-Reformen und der Ausbildung moderner Staatlichkeit (s. Haug 2016, 193). 


\section{Die maria-theresianische Zensurkommission}

1749 wurde für die politische Administration der Monarchie eine neue Zentralstelle geschaffen, das Directorium in Publicis et Cameralibus, das nun auch die Zensurorganisation übernahm. Der Vorschlag des Directoriums lautete, dass in einer neu zu schaffenden Bücher-Censurs-Hofcommission die Zensurbefugnis über theologische und philosophische Bücher bei der Universität verbleiben, die übrigen Fächer aber von weltlichen Zensoren betreut werden sollten. Dabei wurde in Rechnung gestellt, dass die Theologie auf dem Buchmarkt noch immer den Ton angab und die Produktion politischer, historischer und juridischer Literatur nach Einschätzung von Zeitgenossen in Österreich denkbar gering war: In Wien waren laut Angaben der Buchdrucker im Jahr 1751 ,,außer fünf oder sechs Geistlichen und etwa ein paar Weltlichen keine anderen Scribenten vorhanden“ (Klingenstein 1970, 144).

Gerard van Swieten, der diese Vorschläge koordinierte und umsetzte, kann als Schöpfer der maria-theresianischen Zensurreform gelten. Er stellt so etwas wie den Archetyp des Zensors in Österreich dar, der noch dem alten, mit dem Ende des 18. Jahrhunderts aussterbenden Stamm der Polyhistoren angehörte. Präsident der Kommission war zunächst Franz Josef Graf Saurau, der nach kurzer Zeit von Johann Graf Chotek abgelöst wurde; die Fächer Philosophie und Theologie wurden, wie vorgesehen, von den Jesuiten betreut; um die Jurisprudenz kümmerten sich zwei Professoren der juridischen Fakultät der Universität Wien (Ignaz Aigner und Johann Adam Penz); im Fach Medizin zensierte Gerard van Swieten, der 1759 auch den Vorsitz der Kommission übernahm, die historisch-politischen Schriften und das öffentliche Recht wurden von Professoren der Savoyschen und Theresianischen Akademie abgedeckt (Christian August Beck, Paul Joseph Riegger und Johann Heinrich Gottlob Justi) (vgl. Klingenstein 1970, 161 u. Hadamowsky 1979, 290). Van Swieten gelang es bald, sich zusätzlich zum medizinischen Fach auch die philosophischen Schriften und die materies mixtae (in etwa: schöne Literatur) aus dem Kompetenzbereich der Jesuiten zu sichern. Überdies machte er sich erfolgreich über die Praxis der Jesuiten lustig, in Schriften zur Anatomie „Nuditäten“ zu beanstanden, und übernahm in der Folge auch die Zensur der naturwissenschaftlichen Bücher. 1764 wurde der letzte verbliebene Jesuit aus der Kommission verdrängt. Die jesuitischen Mitglieder wurden zwar durch Untergebene des Erzbischofs ersetzt, die laizistisch-staatliche Fraktion hatte im Kampf um die Zensurhoheit dennoch einen wichtigen Sieg davongetragen. Wie van Swieten betonte, konnte der Wiener Erzbischof zwar die geistlichen Mitglieder vorschlagen, sie mussten aber von der Kaiserin bestätigt werden (Fournier 1876, 462).

Im Zeichen der Aufklärung sollte die Zensur insbesondere Ignoranz und Aberglauben zurückdrängen. „Auch konnten mit Hilfe der Zensur die alten Formen von Sitten und Gebräuchen verändert werden, die in den Augen der Aufklärer derb und roh schienen.“ So diente die Zensur ,der Verbreitung einer modernen, 
rigoroseren Moral und der Verfeinerung der Umgangsformen." (Klingenstein 1973, 104) Was als reiner Idealismus im Sinn der Veredelung der Menschheit erscheinen mag, diente auch handfesten Interessen: Der moderne Staat benötigte mündige, selbstständige und vor allem gut informierte Bürger und ökonomische Subjekte. So wurde ein gemäßigter Reformkatholizismus (der Jansenismus) toleriert bzw. gefördert, die Schriften der Jesuiten wurden dagegen ab 1759 verboten, zumal man ihnen unterstellte, dass sie den Fürstenmord billigten. Charakteristisch für den schwindenden Einfluss der Jesuiten ist der Eklat um Montesquieus Esprit des lois (1748). Die Jesuiten hatten das Werk 1750 verboten und bekämpften es weiterhin in der Zensurkommission, deren Mitglieder befürworteten aber mehrheitlich seine Zulassung. Auch der Verfasser, der seit seinem Besuch in Wien intensive Kontakte zu einflussreichen Persönlichkeiten in der Stadt unterhielt, intervenierte in eigener Sache. Er schrieb dem französischen Gesandten in Wien, dass ein Verbot in Wien angesichts des großen Prestiges des Wiener Hofes unter Maria Theresia der Wirkung seines Werks großen Schaden zufügen würde (s. Schmidt 1931, 83-84). Schließlich entschied die Kaiserin, wenn auch mit Verspätung, 1752 im Sinn der Mehrheit der Kommission.

Mit der Neuorganisation der Zensur wurde auch den Bücherverbrennungen von staatlicher Seite ein Ende gesetzt. Fortan ging es nur noch ganz sachlich um die ,Vertilgung' der verbotenen Bücher. Mit der rituellen öffentlichen Verbrennung durch den Henker ist es in Zeiten der vordringenden Aufklärung und Rationalisierung aller Lebensbereiche aber vorbei.

Van Swieten führt in seiner in französischer Sprache verfassten Denkschrift von 1772 die wichtigsten Zensurmotive an (zit. in Bachleitner 2017, 421-427). Ausgangspunkt ist die Feststellung, dass sich die schädlichen Schriften (livres pernicieux) rasch vermehrt hätten. Im Bereich der Religion habe der Deismus an Boden gewonnen, die Protestanten stellten die Autorität des Papstes infrage, der Ablass werde gepredigt, der Aberglaube blühe und die Jesuiten verkündeten die absolute Macht des Papstes über alle Gläubigen und deren Eigentum, einschließlich jenes der weltlichen Herrscher. Von Protestanten verfasste wissenschaftliche Bücher könnten dagegen von großem Nutzen sein und sollten trotz gelegentlicher anti-katholischer Invektiven toleriert werden. Ein glaubensfestes katholisches Publikum sei dadurch nicht ins Wanken zu bringen, im Übrigen kämen die geeigneten Antworten darauf von der Kontroverstheologie. Selbstverständlich müssten unsittliche Bücher (livres impudiques) und Abbildungen kategorisch unterdrückt werden. Ein besonderes Anliegen Van Swietens war der Jugendschutz. Seine Ausführungen bringen die Widersprüche zwischen Apologie und Verurteilung der Zensur und die damit verbundenen Selbstwidersprüche zum Ausdruck, in die sich Aufklärer verwickelten, wenn Sie über Zensur sprachen; sie finden sich in ähnlicher Form bei so prominenten Exponenten der Aufklärung wie Leibniz, Christian Wolff, Gottsched und Kant (vgl. Haefs 2015, 561). 
Van Swieten blieb bis zu seinem Tod im Juni 1772 Präsident der Kommission. Wie gesagt, zensierte er die Belletristik, und das beinahe im Alleingang. Werke von berühmten Autoren wie Ariosto, Machiavelli, Lessing, Wieland, Fielding, Crébillon, Rousseau und Voltaire fanden keine Gnade vor seinen Augen. Van Swieten soll Rousseau gegenüber Friedrich Nicolai anlässlich des Romans Émile sogar als „mauvais sujet“ bezeichnet haben (Nicolai 1784, 854). Voltaire rächte sich für die zahlreichen Verbote seiner Werke mit Spottversen auf Van Swieten, die in der Epitre au roi de Danemarck VII. sur la liberté de la presse accordée dans tous ses états (1771) enthalten waren. Voltaire bezeichnet ihn dort als von Hippokrates abgefallenen Scharlatan, der zwar Kranke, aber niemals gute Bücher umzubringen vermöge.

\footnotetext{
Un certain charlatan, qui s'est mis en crédit,

Prétend, qu'à son exemple, on n'ait jamais d'esprit.

Tu n'y parviendras pas, apostat d'Hippocrate:

Tu guérirais plutôt les vapeurs de ma rate.

Va, cesse de vexer les vivans et les morts;

Tyran de ma pensée, assassin de mon corps,

Tu peux bien empêcher les malades de vivre,

Tu peux les tuer tous, mais non pas un bon livre.

Tu les brûles, Jérôme; et de ces condamnés

La flamme m'en éclairant, noircit ton vilain nez. (Zit. nach Fournier $1876,425)$

(Ein gewisser Scharlatan, der sich Einfluß verschafft hat, gibt vor, daß nur er Geist besitze. Dazu wirst du nicht gelangen, Abtrünniger des Hippokrates; du könntest eher die Dünste meiner Milz heilen. Geh', höre auf, die Lebenden und die Toten zu quälen; Tyrann meines Denkens, Mörder meines Körpers, du kannst wohl deine Kranken am Leben hindern, du kannst sie alle töten, nicht aber ein gutes Buch; du verbrennst sie, Jérome, und die Flamme dieser Verurteilten - mich beleuchtend - schwärzt deine Schurkennase.) (Zit. nach Rafetseder 1988, 244)
}

Van Swieten verachtete die Belletristik, er fand schöngeistige Werke unnütz, oft sogar bösartig, skandalös und gottlos (vilains, scandaleux, impies), was durchaus speziell auf Voltaire gemünzt sein könnte. Aus diesem Grund bedauerte er die Mühe, die er für die Lektüre solcher Schriften aufwenden musste, zumal man daraus keinen bleibenden Gewinn ziehen konnte.

Die Gutachten Van Swietens, die die Grundlage für die Beurteilungen durch die Kommission darstellten, sind in einem Codex gesammelt, der zum Leidwesen der Forschung in einer nur schwer zu entziffernden Kurzschrift verfasst ist (s. Van Leersum 1906). Der Grund für die Verwendung der Kurzschrift mag die Geheimhaltung der Kommentare gegenüber anderen - insbesondere den geistlichen - Kommissionsmitgliedern gewesen sein. Van Swietens Aufzeichnungen betreffen 3.120 Werke, von denen 595, also ein knappes Fünftel, mit ,damnatur 
beurteilt wurden. Auf Betreiben Josephs von Sonnenfels wurde 1770 auch die Zensur von Theatertexten in die Agenden der Kommission aufgenommen. Nach Sonnenfels übernahm dieses Fach der niederösterreichische Regierungsrat Franz Karl Hägelin, der 1795 auch detaillierte Richtlinien für die Zensur von Theaterstücken entwarf (s. Bachleitner 2017, 438-462).

Die Kommission trat einmal monatlich, bei Bedarf auch öfter, bei Van Swieten zusammen (vgl. Abbildung 2). Die Mitglieder referierten über die ihnen zur Beurteilung zugesandten noch unbekannten Bücher, die über das Zollamt in das Bücherrevisionsamt gelangt waren. Eventuell las man einzelne fragliche Stellen aus den Schriften vor, dann wurde über die Beurteilung abgestimmt. Bei einstimmigem Urteil war der Fall erledigt, Verbote wurden an die Kaiserin (de facto die Hofkanzlei) zur Bestätigung weitergeleitet. Bei unterschiedlichen Urteilen wurde der Fall vertagt, alle Zensoren mussten die fragliche Schrift lesen und sich eine Meinung bilden. Wenn dann noch immer keine einhellige Beurteilung erzielt werden konnte, wurden alle Meinungen zu Protokoll genommen und dieses ebenfalls der Kaiserin zur Entscheidung übergeben. Die verbotenen Titel wurden in Form von etwa monatlich zusammengestellten Consignationen in die Provinzen versandt und am Jahresende als Nachtrag zum Catalogus librorum prohibitorum zusammengefügt. In einem merkwürdigen Ritual wurden in den Kommissionssitzungen zudem die bei Privatpersonen beschlagnahmten verbotenen Bücher ,von sammentlichen denen Censoribus und ihme [dem Sekretär der Kommission] sogleich in Stücke zerrissen und vertilget." (Fournier 1876, 419) Nur theologische und politische Literatur wurde, sofern noch nicht vorhanden, in die kaiserliche bzw. die erzbischöfliche Bibliothek inkorporiert.

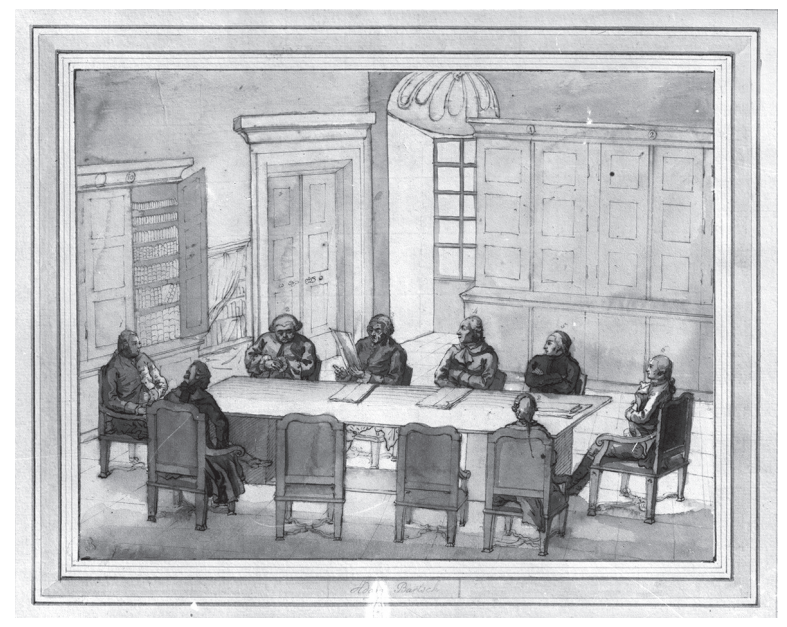

Abbildung 2:

Eine Sitzung bei Gottfried van Swieten in der Camera praefecti. Zeichnung von Adam Bartsch (Österreichische Nationalbibliothek, Bildarchiv und Grafiksammlung) 
Die Information über das Verbot eines bestimmten Buches war bis zur Gründung der Zensurkommission von Fall zu Fall in Form einzelner Verordnungen verbreitet worden. Dieses Verfahren war schwerfällig und führte zwangsläufig zu Informationslücken. Es war geeignet, solange der Buchmarkt klein und überschaubar blieb. Um die genannten Unzulänglichkeiten zu beseitigen, wurde 1754 der laufend ergänzte und aktualisierte Catalogus librorum prohibitorum eingeführt. Für den Zeitraum 1751 bis 1780 konnten 4701 Verbote ermittelt werden, was pro Jahr durchschnittlich 157 verbotene Titel ergibt (s. Bachleitner 2017, 73-74). Dass der Catalogus selbst verboten war, wie oft behauptet wurde, ist nirgendwo belegt und darf folglich bezweifelt werden.

In die 1760er Jahre reicht die Praxis zurück, zwischen den Gebildeten bzw. Oberschichten und dem großen Publikum zu unterscheiden. Am 4. Oktober 1766 verkündete ein Hofdekret, dass fortan verbotene Bücher, die nur einige wenige anstößige Sätze enthielten, gebildeten Lesern genehmigt werden sollten (s. Lavandier 1993, 90). Angehörige höchster Gesellschaftskreise hatten es meist gar nicht nötig, Scheden zu beantragen, sie benützten informelle Kanäle. So soll sich Graf Karl Zinzendorf, wie er in seinem Tagebuch vermerkt, während seiner Zeit als Gouverneur von Triest, das heißt zwischen 1777 und 1780, kistenweise verbotene Bücher aus Frankfurt, Leipzig und per Schiff über Marseille haben liefern lassen (vgl. Wagner 1963, 148).

Am 21. März 1772 wurde die alte, aus Mitgliedern der Universität, des Magistrats und des bischöflichen Consistoriums zusammengesetzte, unter Vorsitz des Wiener Erzbischofs agierende „Bücher-Censur-Commission“ aufgelöst und eine neue Zensurkommission eingerichtet, die als reines Beamtenkollegium konzipiert war. Die neue, bis 1781 agierende Kommission umfasste 12 Mitglieder. Auch theologische Manuskripte mussten nun die weltlich-staatliche Zensur durchlaufen. Der Erzbischof protestierte, wurde aber abgewiesen. Fortan übte er „Nachcensur und legte das Resultat seiner Mühe stets in der umfangreichsten Weise der Regierung, in der Regel dem Cabinete vor.“ (Wiedemann 1873, 296) Das Archiv der Erzdiözese Wien ist voll von Protesten wegen zugelassener a-religiöser oder nicht-dogmatischer Werke und umgekehrt wegen nicht zugelassener, nach Ansicht des Bischofs aber geeigneter Literatur.

Das Zensorenamt wurde nun auch professionalisiert und damit der Usus beendet, nach dem die Zensoren ihre Tätigkeit rein ehrenamtlich ausführten. Künftig erhielten die Kommissionsmitglieder Zulagen bzw. Besoldungen in der Höhe von 300 bis 500 Gulden (Fournier 1876, 446). Der Plan geht aber noch, wie fast alle Reformen der Zensurorganisation, auf deren spiritus rector Van Swieten zurück. In einem Brief vom 24. Februar 1772 an die Kaiserin hatte er, der genau wusste, wovon er sprach, die große Mühe betont, die die Zensur erforderte, und eine angemessene Belohnung für die Zensoren angeregt (Fournier 1876, 464). 


\section{Statistik der Verbotstätigkeit 1754-1791}

In der folgenden Tabelle repräsentieren die Teilsummen jeweils die gesammelten Verbote der einzelnen Jahrzehnte, die ersten drei Teilsummen zusammen die maria-theresianische, die vierte Teilsumme die josephinische und leopoldinische Epoche. Es zeigen sich nur geringfügige Unterschiede zwischen den drei ersten Jahrzehnten der Regierungszeit Maria-Theresias; in der josephinisch-leopoldinischen Ära sank die Verbotstätigkeit um beinahe zwei Drittel, sie betrug nur noch $37 \%$ jener der drei vorangegangenen Jahrzehnte.

Tabelle 1:

Anzahl der Verbote 1754-1791

(Quelle: Verpönt, Verdrängt - Vergessen?)

\begin{tabular}{|c|c|c|}
\hline Jahr & Verbote & Teilsummen \\
\hline 1754 & 669 & \\
\hline 1755 & 393 & \\
\hline 1756 & 197 & \\
\hline 1757 & 191 & \\
\hline 1758 & 118 & \\
\hline 1759 & 158 & 1726 \\
\hline 1762 & 411 & \\
\hline 1763 & 150 & \\
\hline 1764 & 118 & \\
\hline 1765 & 166 & \\
\hline 1766 & 146 & \\
\hline 1767 & 94 & \\
\hline 1768 & 122 & \\
\hline 1769 & 188 & 1395 \\
\hline 1770 & 132 & \\
\hline 1771 & 196 & \\
\hline 1774 & 578 & \\
\hline 1776 & 164 & \\
\hline 1777 & 132 & \\
\hline 1778 & 155 & \\
\hline 1780 & 223 & 1580 \\
\hline 1783 & 5 & \\
\hline 1784 & 267 & \\
\hline 1785 & 47 & \\
\hline 1786 & 36 & \\
\hline 1787 & 42 & \\
\hline 1788 & 37 & \\
\hline 1789 & 54 & \\
\hline 1790 & 68 & \\
\hline 1791 & 85 & 641 \\
\hline Summe & 5342 & 5342 \\
\hline
\end{tabular}


Dass die Zensur im zeitlichen Verlauf tendenziell eher milder verfuhr, ergibt sich daraus, dass sich die deutsche Bücherproduktion zwischen 1760 und 1780 ungefähr verdoppelte.

Die Aufgliederung der Verbote nach Sprachen ermöglicht einen Einblick in die internationalen Verbindungen des Buchhandels und den Kulturtransfer im Berichtszeitraum.

Tabelle 2:

Aufteilung der Verbote 1754-1780 nach Sprachen

(Quelle: Verpönt, Verdrängt - Vergessen?)

\begin{tabular}{lrl}
\hline Deutsch & 2203 & $(=46,8 \%)$ \\
Französisch & 1506 & $(=32,0 \%)$ \\
Lateinisch & 619 & $(=13,2 \%)$ \\
Italienisch & 164 & $(=3,5 \%)$ \\
Englisch & 111 & $(=2,4 \%)$ \\
Andere & 98 & $(=2,1 \%)$ \\
Summe & 4701 & $(=100,0 \%)$ \\
\hline
\end{tabular}

Auffällig ist hier, wie auch Diagramm 1 verdeutlicht, dass das Französische dem Deutschen an Bedeutung zumindest nahe kommt. Es überrascht wenig, dass ferner das Lateinische als gelehrte und Kirchensprache noch stark präsent ist, wohl aber, dass das Englische, das zusammen mit dem Französischen wesentliche Literatur der Aufklärung bereithielt, eine derart untergeordnete Rolle spielt und klar hinter dem Italienischen rangiert.

Tabelle 3: Meistverbotene Autoren 1754-1780

(Quelle: Verpönt, Verdrängt - Vergessen?)

\begin{tabular}{ll}
\hline 1. Voltaire & 92 \\
2. Argens, Jean-Baptiste de Boyer d' & 24 \\
3. Dorat, Claude Joseph & 17 \\
Friedrich II. & 17 \\
Meier, Georg Friedrich & 17 \\
6. Rétif de La Bretonne, Nicolas-Edme & 16 \\
Rousseau, Jean-Jacques & 16 \\
Wieland, Christoph Martin & 16 \\
9. Crébillon, Claude Prosper Jolyot de & 15 \\
10. Leti, Gregorio & 13 \\
\hline
\end{tabular}


Betrachtet man die Liste der am häufigsten verbotenen Autoren, so wird die große Bedeutung des Französischen bestätigt. Unter den ersten zehn Namen finden sich sechs Franzosen, drei Deutsche, von denen einer (Friedrich II.) ebenfalls häufig Französisch schrieb, und ein Italiener. Dass Voltaire an der Spitze liegt, mag nicht überraschen, dass er mit so großem Abstand die Spitzenposition behauptet, aber schon. Der Marquis d'Argens, wie Voltaire Langzeitgast am Preußischen Hof, passt mit seinen philosophischen und belletristischen Werken ganz in das aufklärerische Szenario. Beider ,Dienstgeber', der preußische Philosophenkönig, ist mit philosophischen, historischen und belletristischen Schriften vertreten und rangiert ex aequo mit G. F. Meier, einem weiteren Philosophen, der den Schwerpunkt auf Ästhetik und Religionskritik legte, nur knapp dahinter. Als Konservativer und Anti-Aufklärer fällt Claude Joseph Dorat mit seinen Theaterstücken und Prosaschriften hier als einziger aus dem Rahmen. Rousseau, der zusammen mit Voltaire im deutschsprachigen Raum wohl bekannteste Vertreter der französischen Aufklärung, und die Verfasser satirischer sowie frivol-libertinistischer Prosa bzw. Epik Crébillon fils und Wieland runden das Bild der unter den top ten versammelten aufklärerischen Prominenz ab. Der gebürtige Italiener Gregorio Leti machte als Historiker am französischen und englischen Hof Karriere, seine papst- und kirchenkritischen Schriften standen sämtlich auf dem römischen Index; unter Kollegen galt er - zu Recht - als extrem unzuverlässiger Historiograph.

Die übrigen, hier nicht abgedruckten Namen auf der Liste der ,Spitzenreiter' sind größtenteils weitere Speerspitzen der Aufklärung, sei es auf philosophisch-religionskritischem oder belletristisch-satirischem Gebiet. Wenige Ausnahmen weisen ins 17. Jahrhundert zurück (genannt seien Martin von Cochem, Ferrante Pallavicino, Johannes Praetorius, Johann Beer und Jakob Böhme), die als Protestanten, Satiriker oder Vertreter des Aberglaubens verpönt wurden. Aus der Renaissanceliteratur ist Aretino vertreten, aus der Antike lediglich Ovid, beide sind wegen ihrer Erotika unter die Verbots-Spitzenreiter geraten. Wenn die Zensur im behandelten Zeitraum ganz allgemein die Aufklärung unterstützt, so treten hier die Grenzen der Toleranz gegen die radikalen Ausläufer dieser Strömung sehr deutlich hervor.

\section{Quellen}

Bachleitner, Norbert, Die literarische Zensur in Österreich von 1751 bis 1848. Mit Beiträgen von Daniel Syrovy, Petr Píša und Michael Wögerbauer (Wien, Köln, Weimar: Böhlau, 2017).

Ducreux, Marie-Elisabeth, "Reading unto Death. Books and Readers in Eighteenth-Century Bohemia," in The Culture of Print. Power and the Uses of Print in Early Modern Europe, ed. by Roger Chartier (Princeton, N. J.: Princeton University Press, 1989), pp. 191-229. 
Fischer, Heinz-Dietrich (Hg.), Deutsche Kommunikationskontrolle des 15. bis 20. Jahrhunderts (München, New York, London, Paris: Saur, 1982).

Fournier, August, "Gerhard van Swieten als Censor", Sitzungsberichte der Kaiserlichen Akademie der Wissenschaften. Philosophisch-historische Klasse 84 (1876), Heft 3, pp. 387-466.

Hadamowsky, Franz, "Ein Jahrhundert Literatur- und Theaterzensur in Österreich (1751-1848)", in Die Österreichische Literatur. Ihr Profil an der Wende vom 18. zum 19. Jahrhundert (17501830) (Graz: Akademische Druck- und Verlagsanstalt, 1979), Teil 1, pp. 289-305.

Haefs, Wilhelm, Art. "Zensur", in Handbuch Europäische Aufklärung. Begriffe - Konzepte Wirkung (Stuttgart, Weimar: Metzler, 2015), pp. 558-567.

Haug, Christine, “'Literatur aus dem Giftschrank' - Kontexte und Mythen. Buchmarkt und zensurpolitische Strategien im literarischen Untergrund im Zeitalter der Aufklärung. Ein Forschungsbericht", Archiv für Geschichte des Buchwesens 71 (2016), pp. 185-226.

Klingenstein, Grete, Staatsverwaltung und kirchliche Autorität im 18. Jahrhundert. Das Problem der Zensur in der theresianischen Reform (Wien: Verlag für Geschichte und Politik, 1970).

Klingenstein, Grete, "Van Swieten und die Zensur", in Gerard van Swieten und seine Zeit, hg. v. Erna Lesky u. Adam Wandruszka (Wien, Köln, Graz: Böhlau, 1973), pp. 93-106.

Lavandier, Jean-Pierre, Le livre au temps de Marie-Thérèse. Code des lois de censure du livre pour les pays austro-bohémiens (1740-1780) (Bern, Berlin, Frankfurt/Main, New York, Paris, Wien: Peter Lang, 1993).

Nicolai, Friedrich, Beschreibung einer Reise durch Deutschland und die Schweiz im Jahre 1781. Bd. 4. (Berlin, Stettin: Nicolai, 1784).

Rafetseder, Hermann, Bücherverbrennungen. Die öffentliche Hinrichtung von Schriften im historischen Wandel (Wien, Köln, Graz: Böhlau, 1988).

Scheutz, Martin, "Das Licht aus den geheimnisvollen Büchern vertreibt die Finsternis. Verbotene Werke bei den österreichischen Untergrundprotestanten", in Kriminelle - Freidenker - Alchemisten. Räume des Untergrunds in der Frühen Neuzeit, hg. v. Martin Mulsow (Köln, Weimar, Wien: Böhlau, 2014), pp. 321-351.

Schmidt, Justus, "Voltaire und Maria Theresia. Französische Kultur des Barock in ihren Beziehungen zu Österreich", Mitteilungen des Vereines für Geschichte der Stadt Wien 11 (1931), pp. $73-115$.

Van Leersum, E. C., "Gérard van Swieten en qualité de censeur", Janus. Archives internationales pour l'Histoire de la Médecine et la Géographie Médicale 11 (1906), pp. 381-398, 446-469, 501-522, 588-606.

Verpönt, Verdrängt - Vergessen? Eine Datenbank zur Erfassung der in Österreich zwischen 1750 und 1848 verbotenen Bücher (http://www.univie.ac.at/zensur).

Wagner, Hans, "Historische Lektüre vor der Französischen Revolution - aus den Tagebüchern des Grafen Karl von Zinzendorf, Mitteilungen des Instituts für österreichische Geschichtsforschung 71 (1963), pp. 140-156.

Wiedemann, Theodor: Die kirchliche Bücher-Censur in der Erzdiöcese Wien. Nach den Acten des Fürsterzbischöflichen Consistorialarchives in Wien. In: Archiv für Kunde österreichischer Geschichtsquellen 50 (1873), S. 215-520.

\section{Anmerkungen}

1 Institut für Europäische und Vergleichende Sprach- und Literaturwissenschaft, Abteilung für Vergleichende Literaturwissenschaft, Sensengasse 3a, A-1090 Wien, email: norbert.bachleitner@univie.ac.at. 